\title{
Bright trions in direct-bandgap silicon nanocrystals revealed by low-temperature single-nanocrystal spectroscopy
}

\begin{abstract}
Kateřina Kůsová ${ }^{1}$, Ivan Pelant ${ }^{1}$ and Jan Valenta ${ }^{2}$
Strain-engineered silicon nanocrystals (SiNCs) have recently been shown to possess direct bandgap. Here, we report the observation of a rich structure in the single-nanocrystal photoluminescence spectra of strain-engineered direct-bandgap SiNCs in the temperature range of 9-300 K. The relationship between individual types of spectra is discussed, and the numerical modeling of spectral diffusion of the experimentally acquired spectra reveals a common origin for most types. The intrinsic spectral shape is shown to be a structure that contains three peaks, approximately 150 meV apart, each of which possesses a Si phonon substructure. Narrow spectral lines, reaching $\leqslant 1 \mathrm{meV}$ at $20 \mathrm{~K}$, are detected. The observed temperature dependence of the spectral structure can be assigned to the radiative recombination of positively charged trions, in contrast to several previous reports linking a very similar shape to phonons in the surface capping layers. Our result serves as strong additional support for the direct-bandgap nature of the investigated SiNCs.
\end{abstract}

Light: Science \& Applications (2015) 4, e336; doi:10.1038/Isa.2015.109; published online 09 October 2015

Keywords: direct bandgap; low-temperature single-nanocrystal spectroscopy; silicon nanocrystals; trions

\section{INTRODUCTION}

Bulk silicon, as an indirect-bandgap semiconductor, is a poor light emitter and is thus unsuitable for active photonic applications. Silicon nanocrystals (SiNCs), by contrast, exhibit luminescence at room temperature (RT). The use of SiNCs instead of the bulk form of silicon, however, can alleviate the problem of poor light emission only partly, because SiNCs preserve the indirect nature of the bandgap ${ }^{1,2}$, and the light-emission rate thus remains low, on the order of $10^{4} \mathrm{~s}^{-1}$ or less ${ }^{3}$. However, when passivated by a "suitable" organic material, SiNCs can have fast macroscopic radiative lifetime ${ }^{4-7}$ and can actually be transformed into a direct-bandgap material ${ }^{5,8-10}$. In particular, we have recently shown both theoretically and experimentally that properly capped SiNCs can be strain-engineered into a material with fundamental direct $\Gamma_{15}-\Gamma_{25}$ ' bandgap ${ }^{5}$ and the corresponding fast electron-hole radiative recombination rate of $10^{8} \mathrm{~s}^{-1}$ (see also Figure 1a and the section on "Results overview").

The light-emission properties of inherently direct-bandgap semiconductor nanocrystals (NCs) on both the single-NC and the macro level are now well understood ${ }^{11,12}$, even though new phenomena can still be unraveled. For example, the process of non-radiative Auger recombination has, in some cases, been successfully suppressed to a large extent (e.g., Refs. 13, 14), thereby enabling the detection of trions with reasonably high emission efficiency. ${ }^{13,15,16}$ The radiative recombination of a trion has also been proposed to play an important role in blinking ${ }^{17}$.
With respect to single-NC spectroscopy in SiNCs, several types of samples have already been probed by different groups ${ }^{3,4,6,18-27}$. The most commonly observed spectral shape was a mixture of broader unstructured spectra and structured spectra with peaks approximately $150 \mathrm{meV}$ apart. Although the spectral structure looks almost identical in all the measurements, no clear agreement on the mechanism behind it exists. Some groups have suggested that this structure arises due to the involvement of surface-related $\mathrm{SiO}_{2}{ }^{18,22}$ or $\mathrm{SiC}$ phonons ${ }^{6,22}$. In contrast to the phonon-based explanation, our group noted not only the similarities of the spectral structures observed in SiNCs prepared by various methods but also the similarity of the spectral structures observed in SiNCs and II-VI NCs ${ }^{13}$. Therefore, we $\operatorname{argued}^{24}$ that this structure is more closely connected to the core of the NC rather than to its surface.

This article is an in-depth study of single-NC spectroscopy of strainengineered direct-bandgap SiNCs, performed over a broad temperature range of 9-300 K. The observed spectra are rich in spectral structure, and our analysis, which takes into account the calculated bandstructure, spectral diffusion (SD) and electron-phonon interaction, produces an interpretation in terms of the radiative recombination of positively charged trions. This interpretation is to be contrasted with the commonly held belief that efficient non-radiative Auger recombination in silicon prevents the possibility of the observation of luminescence from electron-hole complexes, which contain more than a single electron-hole pair. Moreover, it serves as additional proof of the feasibility of rendering silicon a material with

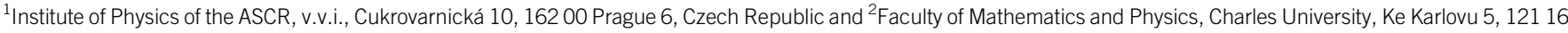
Prague 2, Czech Republic 

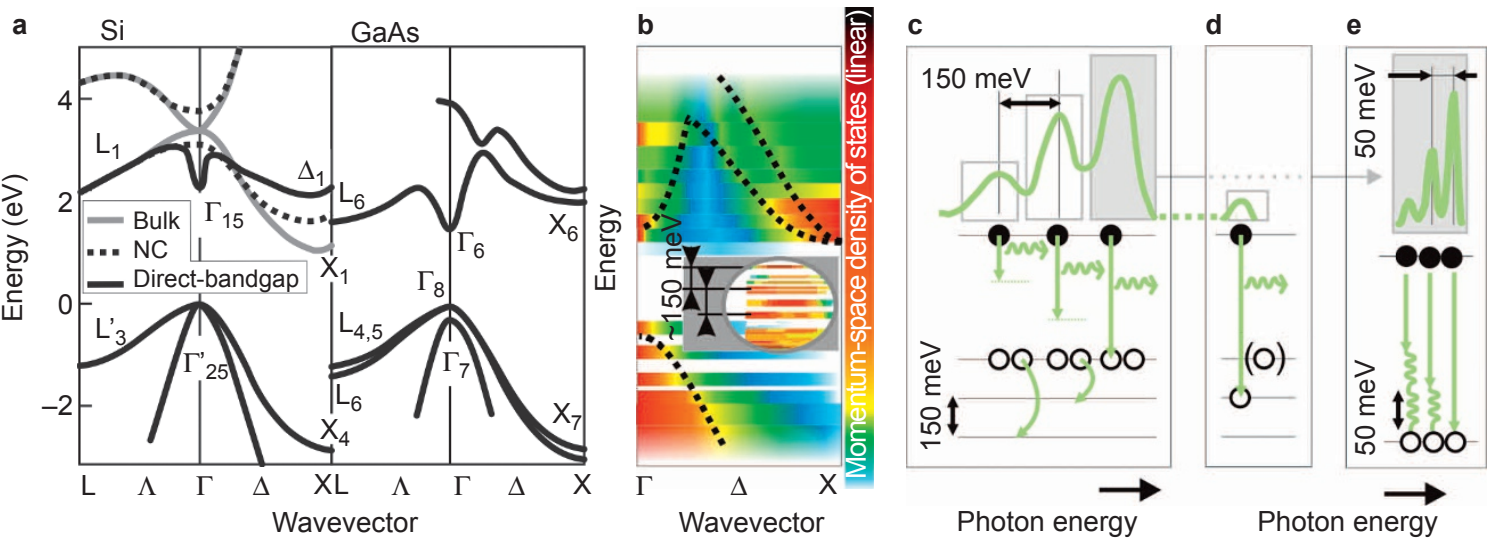

Figure 1 Sketch of the bandstructure of the studied SiNCs and trionic recombination. (a) Direct comparison of bandstructures of the investigated SiNCs and a traditional example of direct-bandgap material. (b) The colored areas represent the projection of the electron and hole wavefunctions to $k$-space (calculated bandstructure of the SiNCs under study). The black dotted curve highlights the band edges in SiNCs and serves as a guide-to-the-eye. The inset shows a detail of the valence-band maximum, with highlighted states approximately $150 \mathrm{meV}$ apart. Modified after Ref. 5. (c) Radiative recombination of a trion. (d) The high-energy wing: a not fully relaxed hole recombines with an electron. (e) The phonon sub-structure; phonon involvement in a recombination process can occur for any of the trionic replicas or the high-energy wing.

fundamental direct bandgap, which makes the commonly (over)used phrase stating that "silicon is an indirect-bandgap material" lose its general validity.

\section{MATERIALS AND METHODS}

The SiNCs under study were, in brief, prepared by repassivating originally indirect-bandgap, oxide-covered SiNCs, which were themselves prepared by electrochemical etching ${ }^{28}$, with methyl-based capping using photochemical treatment in a mixture of aromatic hydrocarbons. Details of the preparation procedure and the investigation of the direct-bandgap switch-over in this material were published elsewhere ${ }^{5,24,29}$. The strain-engineered direct-bandgap SiNCs under study are characterized by short radiative lifetime $\mathrm{e}^{5,24}$ of $10 \mathrm{~ns}$ and a mean SiNC diameter of $2.5-3 \mathrm{~nm}^{24,30}$. Room-temperature single-NC spectroscopy of the oxidized precursor was also performed, but no signs of spectral structure appeared ${ }^{20}$ (see also Supplementary Fig. S1).

The single-NC measurements were performed using a specially designed setup based on an inverted microscope coupled to a $30-\mathrm{cm}$ imaging spectrograph with a back-thinned CCD camera. Samples (colloidal dispersions with appropriate concentration of NCs) were deposited by drop casting on a fused silica substrate and were then placed in the sample compartment. A single objective lens $(100 \times / 0.8$ for room-temperature and $40 \times / 0.6$ for low-temperature measurements) was used to focus the excitation light and to collect and image the emitted signal (epifluorescence configuration). For the low-temperature measurements, the sample was placed in a Janis ST-500 cryostat. Excitation by a 473-nm diode laser was sent to the sample through an optical system, which enabled the excitation of a wide field of a sample. The excitation power density was varied between $\approx 7-80 \mathrm{~W} \mathrm{~cm}^{-2}$ using neutral-density filters. The spatial resolution of the apparatus was restricted by a diffraction limit to approximately $800 \mathrm{~nm}$. All spectra were corrected for the spectral response of the apparatus, and hot-spots were corrected for (by replacing with a neighboring pixel's signal value) in the presented spectra. Overall, 243 room-temperature and 110 low-temperature spectra were studied.

The applied fitting procedures are described in detail in the Supplementary Information (Supplementary Sections S2.3 and S2.5).

\section{RESULTS AND DISCUSSION}

\section{Results overview}

Figure 1 gives a general overview of both the bandstructure of the SiNCs under investigation and the relevant recombination processes. Panel a schematically illustrates how direct bandgap is formed in the NCs under study: starting from indirect bulk Si (the gray curve), the influence of quantum confinement (the dotted curve) leads to the up-shift of the conduction-band minimum $\Delta_{1}$ and a simultaneous down-shift of the conduction-band maximum $\Gamma_{15}$. Tensile strain induced by the methyl capping then further up-shifts the $\Delta_{1}$ minimum and forms a dip in the $\Gamma_{15}$ maximum, thereby transforming methylcapped SiNCs into a direct-bandgap material (the black curve; note the good correspondence with the bandstructure of an example of a direct-bandgap material GaAs on the right). This transformation is supported both experimentally by optical measurements and theoretically by density functional theory calculations, both of which have been published elsewhere ${ }^{5}$. We should stress here that the computed bandstructure of NCs is "fuzzy" due to the Heisenberg uncertainty relations and contains sharp, level-like states separated by minigaps of forbidden energies, especially close to band edges. Panel b shows the fuzzy bandstructure calculated by projecting computed molecular orbitals into $k$-space using the protocol outlined in Ref. 1: the conduction-band minimum is characterized by an isolated electron level, and three groups of energy levels $\sim 150 \mathrm{meV}$ apart are located close to the $\Gamma$ point of the valence band (see the inset). Panel $c$ presents a scheme of the radiative recombination of a trion: ${ }^{13}$ an electron-hole pair can recombine radiatively either by passing all of its energy to the emitted photon (the process on the right) or by transferring a small part of the recombination energy to the remaining hole, thereby raising it to an excited level in the valence band (the processes in the middle and on the left). The corresponding emission spectrum then consists of a series of lines $\sim 150 \mathrm{meV}$ apart with decreasing intensity toward longer wavelengths. Subsequently, the excited hole may recombine with an electron in the conduction-band minimum, giving rise to a weak high-energy wing in the emission spectrum (panel d in Figure 1). Finally, as a result of its excitonic origin, each of the trionic lines can be expected to be composed of phonon replicas, as sketched in Figure 1e. Below, we demonstrate that 

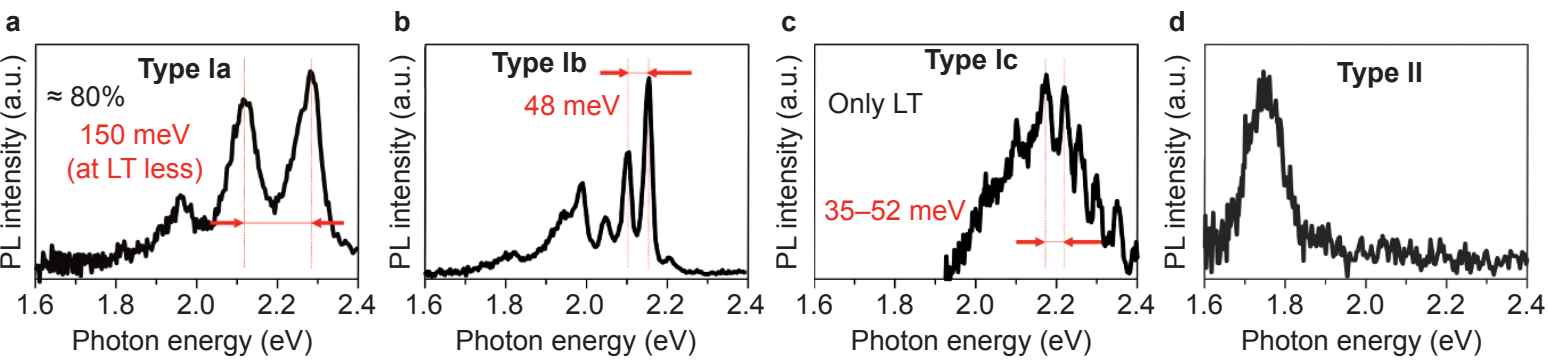

Figure 2 Summary of the types of single-NC spectra observed in the studied samples.

the observed single-NC spectra match the shape outlined above very well.

The single-NC measurements were performed at RT and at lower temperatures (LT) of 150, 60, 40, 20, and 9 K. Figure 2 displays selected examples of the acquired single-NC spectra. Basically, two types of spectral shapes (with three sub-types for the first type) were observed. The first, most commonly encountered spectral shape (approximately $80 \%$ at all temperatures) is that depicted in Figure 2a, which is denoted as type Ia. Its spectral structure is characterized by broader sidebands split-off by $\sim 150 \mathrm{meV}$. The second sub-type, which is denoted as type $\mathrm{Ib}$ (Figure $2 \mathrm{~b}$ ), reveals a sub-structure of very narrow lines that are spaced by $\sim 50 \mathrm{meV}$ and whose intensities are modulated by the broader 150-meV spectral structure. The type-Ib spectral shape was observed for $\sim 10 \%$ of RT measurements. A third sub-type of spectral shape, a type-Ic spectrum, appeared only at low temperatures ( $\leqslant 40 \mathrm{~K}$ ). The representative shape of this type of spectrum is depicted in Figure $2 c$. The type-Ic spectrum is composed of narrower lines that form a broader peak; the lines are better resolvable at the high-energy end of the spectrum. The second main spectral shape, type II, as shown in Figure 2d, generally lacks any structure, tends to be situated at the lower-energy ("red") part of the emission spectrum (especially at RT) and is generally broader, with a Lorentzian lineshape.

Figure 3 illustrates that type-Ib spectra tend to be the narrowest ones: they reach the full width at half maximum (FWHM) of $26 \mathrm{meV}$ at RT (the corresponding $k_{B} T=25.85 \mathrm{meV}$ ) in Figure 3c and $\leqslant 1 \mathrm{meV}$ at $20 \mathrm{~K}\left(k_{B} T=1.7 \mathrm{meV}\right)$ in Figure $2 \mathrm{~b}$. These narrow lines, in combination with the clear blinking shown in Figure $3 \mathrm{a}$, confirm that the observed emission originates in a single NC (see Supplementary Section S2.2 for a discussion of the typical features of single-NC emission for other spectral shapes).

\section{Trionic structure}

Starting with the broader spectral structure split-off by $\sim 150 \mathrm{meV}$, it well reflects the trionic structure schematically represented in Figure 1c. Interestingly, a similar spectral pattern was observed not only in II-VI-based semiconductor $\mathrm{NCs}^{13}$, being interpreted as the emission of a trion, but also by independent groups studying SiNCs, this time being assigned to phonon replicas. In particular, basically identical single-NC spectra with nanosecond dynamics were observed in both nominally $\mathrm{SiO}_{2}$-surface-capped $\mathrm{SiNCs}^{4,22,23}$ and SiNCs capped with $n$-butyl ${ }^{6}$. In these cases, the low-energy satellites were identified with processes that involved the emission of a mixture of $\mathrm{SiO}_{2} \mathrm{TO}$ and LO phonons ${ }^{22}$ and the $\mathrm{Si}-\mathrm{C}$ stretching-vibration phonon $^{6}$, respectively (for an overview of single-NC experiments in SiNCs, see Supplementary Table S1, Fig. S1 and Section S1). Obviously, because more processes can explain the observed line spacing, the energetic distance alone cannot serve as a definitive proof of the origin of the spectral structure.
In the case of the direct-bandgap SiNCs under study, however, the results of temperature-dependent single-NC spectroscopy are clearly not consistent with a phonon-based origin of this spectral structure. First, we showed previously ${ }^{24}$ that these SiNCs are surface-terminated by $-\mathrm{CH}_{3}$ groups, which implies that the occurrence of Si-O-Si phonons at the interface of the NCs at such an abundance is highly unlikely. Second, as follows from Figure 4a, a high-energy wing separated by the same spacing is occasionally observed at RT and becomes more intense with decreasing temperature. Were phonons responsible for this spectral structure instead of trionic recombination, the a
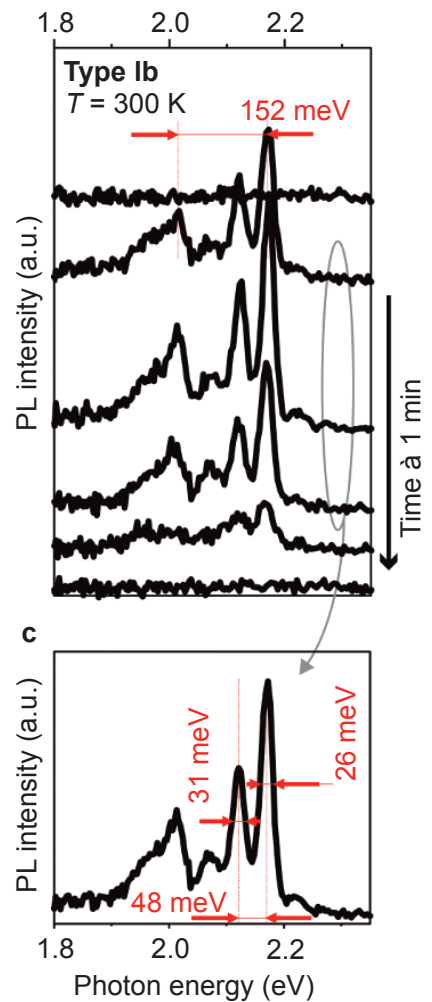

b Photon energy (eV)
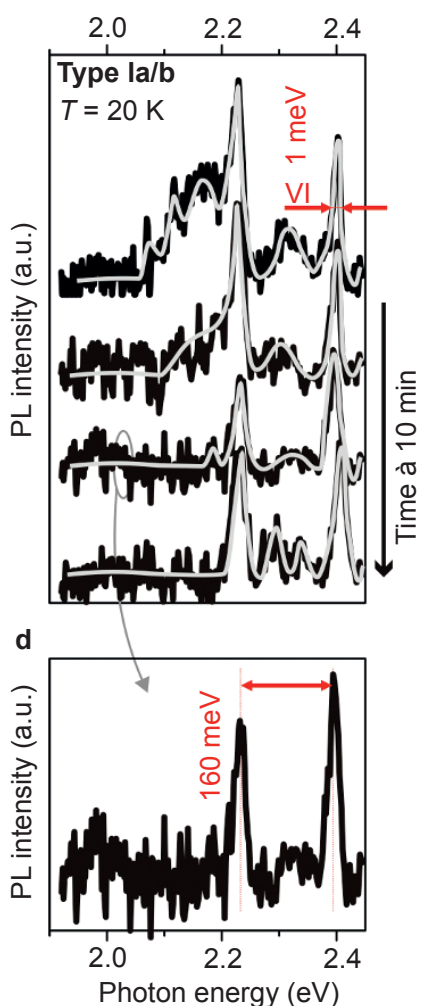

Figure 3 Examples of narrow spectral lines. (a, b) Examples of time series of consecutive single-NC spectra (offset for clarity) acquired at one specific spot. Temperatures are noted in each panel. Panel a illustrates exceptionally narrow type-lb spectra measured at room temperature $\left(k_{B} T=25.85 \mathrm{meV}\right)$. This time series shows blinking, thus confirming that the emission originated in one specific NC. Panel $\mathbf{b}$ presents spectra with the narrowest observed lines $\left(k_{B} T\right.$ at $20 \mathrm{~K}$ is $1.7 \mathrm{meV}$; the linewidth values are deconvolved with the spectral resolution of the system). The gray curves serve as guides-to-the-eye. (c, d) Averages of the spectra from the panels above them designated with the ellipses. 
a

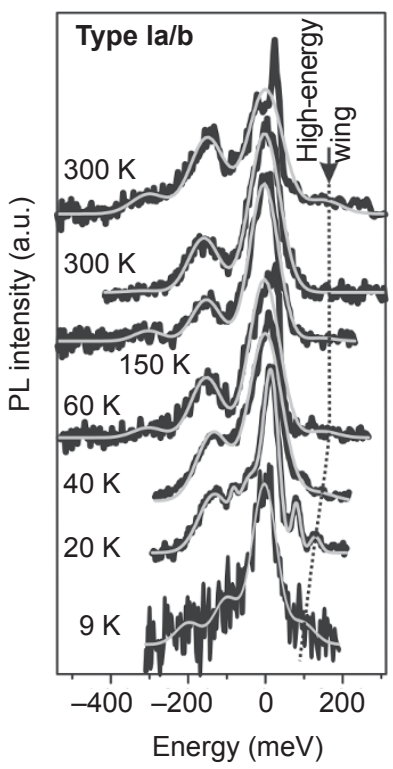

b

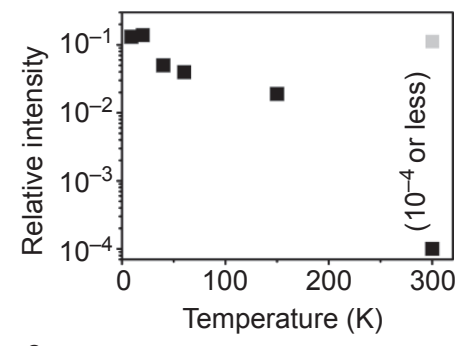

c

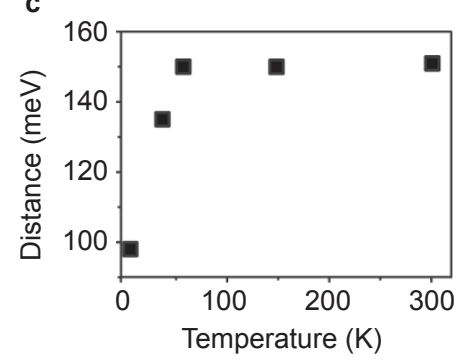

Figure 4 The emergence of the high-energy wing with decreasing temperature. (a) The topmost spectrum is an example of a RT spectrum with exceptionally high intensity of the high-energy wing (for a RT case); the following spectra are measured from different spots at different temperatures with the intensity of the highenergy wing characteristic for the given temperature. Spectra are offset in the photon-energy and intensity axes for clarity, and the gray curves are fits. (b) The fitted area of the high-energy wing with respect to the area of the main peak; the gray square corresponds to the uppermost spectrum in panel a with the intense high-energy wing. (c) The fitted distance between the trionic peaks.

high-energy wing would have to correspond to a process in which a phonon is absorbed. In the case of phonon-assisted transitions in the vicinity of the optical absorption edge in semiconductors, the relative intensities of the processes of phonon absorption and emission $R$ are of the same form as the relationship between anti-Stokes $\left(I_{A S}\right)$ and Stokes $\left(I_{S}\right)$ Raman scattering lines ${ }^{31}$ :

$$
R(T)=\frac{I_{A S}}{I_{S}}=\frac{\text { phonon absorption }}{\text { phonon emission }}=\exp \left(-\frac{\hbar \Omega}{k_{B} T}\right)
$$

where $\hbar \Omega$ is the phonon energy and $T$ stands for temperature. Obviously, this ratio is strongly temperature-dependent: considering $\hbar \Omega \approx 150 \mathrm{meV}$, we obtain $R(300 \mathrm{~K}) \approx 2 \times 10^{-3}$ and $R(20 \mathrm{~K}) \approx 3 \times$ $10^{-40}$. This estimate completely contradicts the behavior observed in the studied SiNCs, where the relative intensity of the high-energy wing actually rises with decreasing temperature, as shown in Figure $4 \mathrm{~b}$, instead of being completely absent. This result confirms that phonons are not involved in the broad-peak trionic structure in the studied NCs.

By contrast, the trion-based explanation fits the observed behavior of the high-energy wing perfectly. When it is connected to a radiative trionic recombination, the high-energy wing originates in the recombination of not completely relaxed (hot) holes; see Figure 1d. Recent theoretical calculations ${ }^{32}$ of multiphonon relaxation rates in SiNCs have predicted that the overall relaxation rate between the first excited and fundamental hole levels in the valence band is strongly, although non-monotonously, size-dependent. At RT, the relaxation rate amounts to $\sim 10^{11} \mathrm{~s}^{-1}$, which translates into the relaxation "decay time" of $\sim 10$ ps. This is a very fast process compared with the photoluminescence (PL) decay time in the SiNCs under study ${ }^{5,24}$

(2 ns), which implies that the high-energy wing should not be observed. However, as a result of the strong size dependence, the relaxation time can change by several orders of magnitude with only a $10 \%$ change in size and can reach values as high as nanoseconds ${ }^{32,33}$. Therefore, certain NCs with suitable sizes are those in which the hothole-related high-energy wing is observed. This strong size dependence also naturally explains the observed very broad distribution of the relative intensity of the high-energy wing described in the Supplementary Information (see Supplementary Fig. S7c and Supplementary Section S2.4), which cannot result from a phonon-based process. Moreover, at low temperatures, the relaxation rate slows down ${ }^{32}$ by a factor of approximately 20 , thereby increasing the relative percentage of hot holes and, consequently, the relative intensity of the hot-hole highenergy wing, again in agreement with our experimental results.

As the observed emission arises from the radiative recombination of a positively charged trion, the peak separation of the high-energy wing, as well as that in the trionic structure, should be characteristic of the energy level splitting at the top of the valence band. The measured value of level separation of $\sim 155 \mathrm{meV}$ at RT agrees reasonably well with the values predicted by the theoretical calculations for $\mathrm{SiNCs}^{32,34}(120 \mathrm{meV})$ and with those observed for $\mathrm{CdZnSe} / \mathrm{ZnSe}$ $\mathrm{NCs}^{13}(164 \mathrm{meV})$. With decreasing temperature, the measured spacing exhibits a slight decrease, as shown in Figures $4 \mathrm{c}$ and $5 \mathrm{a}$, as expected for level separation in a NC, in contrast ${ }^{35}$ to phonons (see Supplementary Section S4).

\section{Phonon sub-structure}

Returning to the finer sub-structure of type-Ib spectra from Figure $2 b$, we should first discuss the relationship between the type-Ia and type-Ib spectral types. Whereas the widths of the narrow sub-structure lines in type-Ib spectra can be very close to temperature- or resolution-limited broadening (see Figure $3 \mathrm{~b}$ and $3 \mathrm{c}$ ), the type-Ia trionic peaks are much broader (100-120 meV). This phenomenon suggests that type-Ia spectra are influenced by non-negligible SD; in other words, random spectral jumps of emission lines from single $\mathrm{NCs}^{11,36-39}$ lead to the smearing of the sub-structure over longer acquisition times (an analogical situation, where approximately $10 \%$ of the studied NCs had narrow spectral lines, occurred, e.g., in Ref. 37). Therefore, the Ib-type spectra represent the "intrinsic" spectral shape, where the influence of SD is minimized.

The assignment of the narrow lines in the type-Ib spectra to a zerophonon line and Si phonon replicas of the corresponding recombination process is quite straightforward-the same spectral shape with phonon replicas and line spacing comparable to bulk phonon frequency is typically observed in direct-bandgap semiconductor $\mathrm{NCs}^{11}$. To support this interpretation, in Figure $5 \mathrm{c}$, we present a series of single-NC Ib-type spectra, measured from the same NC with varying excitation intensity. We fitted the overall spectral shape, assuming that all the trionic peaks share the same (phonon) sub-structure. To do so, the phonon lines were fitted with evenly spaced Gaussian lines with the spacing, widths and intensities shared by all three trionic peaks in all four spectra (because the emission comes from one specific NC). The intensities of the phonon replicas were described using the Huang-Rhys factor $S$, which is known to express the relative intensity $I_{p n}$ of an $n^{\text {th }}$ phonon replica in an optical spectrum as ${ }^{40}$

$$
I_{p n}=\frac{S^{n} \exp (-S)}{n !}
$$

( $n=0$ for the zero-phonon line). The results of this fitting procedure, as presented in the table in Figure $5 \mathrm{~d}$, confirm that the fits (the smooth light-gray curves in Figure 5c) are in excellent agreement with 
a

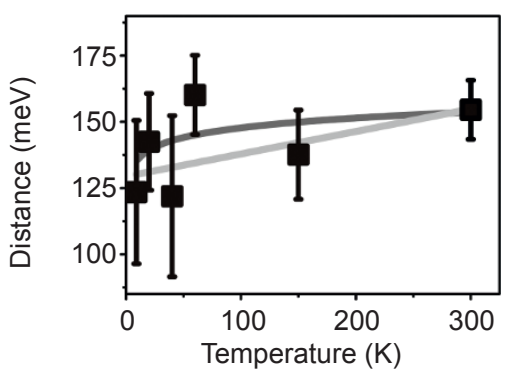

b

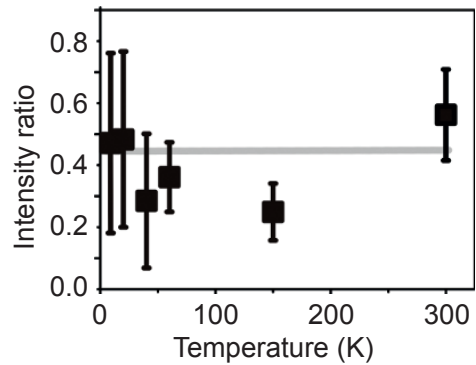

d

\begin{tabular}{|l|lllll|}
\hline$\Delta_{0}(\mathrm{meV})$ & 2.164 & 2.161 & 2.174 & 2.170 \\
$\Delta_{\mathrm{p}}(\mathrm{meV})$ & & ${ }^{4} 48.2$ & & & \\
$\Delta_{\mathrm{t}}(\mathrm{meV})$ & & $* 152$ & & & \\
$I_{\mathrm{t} 2}$ & 0.75 & 1.01 & 0.78 & 0.68 \\
$I_{\mathrm{t} 3}$ & 0.14 & 0.27 & 0.19 & 0.14 \\
$S$ & & ${ }^{*} 0.68$ & & \\
$\omega(\mathrm{meV})$ & 31 & 38 & 24 & 26 \\
$\omega_{\mathrm{p}}(\mathrm{meV})$ & & $* 4.9$ & &
\end{tabular}

c

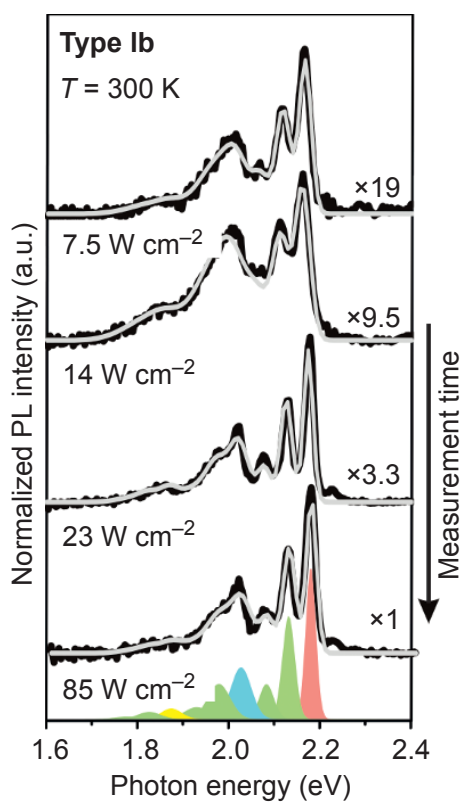

Figure 5 Fits of the observed spectral structures. (a, $\mathbf{b})$ Statistics of the trionic structure: mean distance of the peaks (a) and ratio of the intensities of the first and second trionic peaks (b) are plotted against temperature. The gray lines are guides-to-the-eye highlighting the trends; in panel a, two possible trends are included. (c) Fits (the smooth, light-gray curves) of four Type-lb spectra of one specific NC with increasing excitation intensity, as noted at each spectrum. The spectra are normalized to the same maximum; normalization parameters are listed at each spectrum. The spectra are offset in the intensity axis for clarity; in the bottommost spectrum, the fit is broken down into the individual fitted phonon replicas (red, blue, and yellow for the first, second, and third trionic lines, respectively, and the green lines represent the corresponding one- and two-phonon replicas). (d) The values of fitting parameters; those marked with an asterisk are shared by all four spectra. The inset explains the parameters; $S$ stands for the Huang-Rhys factor.

the measured shapes. Moreover, the conservation of the overall spectral shape, despite the slight shifts of the whole spectrum (see Supplementary Fig. S8a), provides additional proof that all the observed lines originate in the state of one specific NC rather than being a result of the presence of more light-emitting species. (In RT type-Ib spectra, anti-Stokes $\mathrm{Si}$ phonon replicas could also be observed, see Supplementary Section S2.6.)

The fitted value of the phonon energy of $48 \mathrm{meV}$ is lower than the bulk TO phonon energy ( $63 \mathrm{meV}$ at the $\Gamma$ point); however, this is, as we argued in Ref. 5, connected with the influence of the naturally straining methyl capping (see also Supplementary Section S5). We want to note here that the same phonon sub-structure also exists in the high-energy wing, as discussed in Supplementary Section S2.7.

\section{Spectral diffusion and low temperatures}

Moving to the next spectral type, i.e., Ic, an important clue to its physical origin is given by Figure 6a. In this time series, a type-Ia spectrum containing a high-energy wing with resolved phonon sub-structure progressively transforms into a type-Ic spectrum, which suggests a close link between these spectral types. This close link is confirmed by our simulations of SD, where a type-Ib spectrum with a non-negligible highenergy wing is allowed to undergo a series of randomly generated spectral jumps; these diffusing spectra are then summed together to mimic longer acquisition times. The results of these simulations, as shown in Figure $6 \mathrm{c}$, prove that a type-Ib spectrum can be smeared by SD into a type-Ic shape, if the high-energy wing is intense enough. This explains why this spectral type occurs only at low temperatures, where the relative intensity of the high-energy wing increases (see Figure $4 \mathrm{~b}$ ). More examples of simulations of SD, including those confirming the possibility of a type-Ib spectrum being smeared into a type-Ia shape, are included in the Supplementary Information (Supplementary Section S3; note that the amplitudes of spectral jumps assumed in our simulations are in excellent agreement with the values measured in semiconductor $\mathrm{NCs}^{37}$ ). Thus, type Ib, unlike the Ia and Ic shapes, is the "intrinsic" spectral shape that is uninfluenced by SD.

\section{Type-II spectra}

The fourth observed spectral type, type II, is evidently qualitatively different from type-I spectra. We assign this spectral type to NCs with indirect-bandgap-like characteristics because (i) type-II spectra tend to be red-shifted at RT compared with type-I spectra, (ii) they exhibit blue-shift at LT, which is analogical to the one we measured for indirect-bandgap SiNCs macroscopically ${ }^{33}$, and (iii) at LT, their spectral fingerprint is very similar to that observed in indirect-bandgap SiNCs by other groups ${ }^{25,26}$. The possibility of some of the NCs emitting spectra with indirect-bandgap-like characteristics is not as odd as it might seem because (i) our SiNCs were transformed via strainengineering to a direct-bandgap material from an indirect-bandgap precursor, thus implying that some of the non-transformed NCs might have remained in the sample and single-NC spectroscopy allowed us to uncover their presence, and (ii) some of the NCs might have been unintentionally oxidized during the single-NC measurement, thus rendering them indirect. More information is available in the Supplementary Information (Supplementary Section S2.1).

\section{Summary and discussion}

Now, we provide an overview of all the arguments supporting the scenario in which trions are involved in the radiative recombination process in the direct-bandgap SiNCs under study: 
a

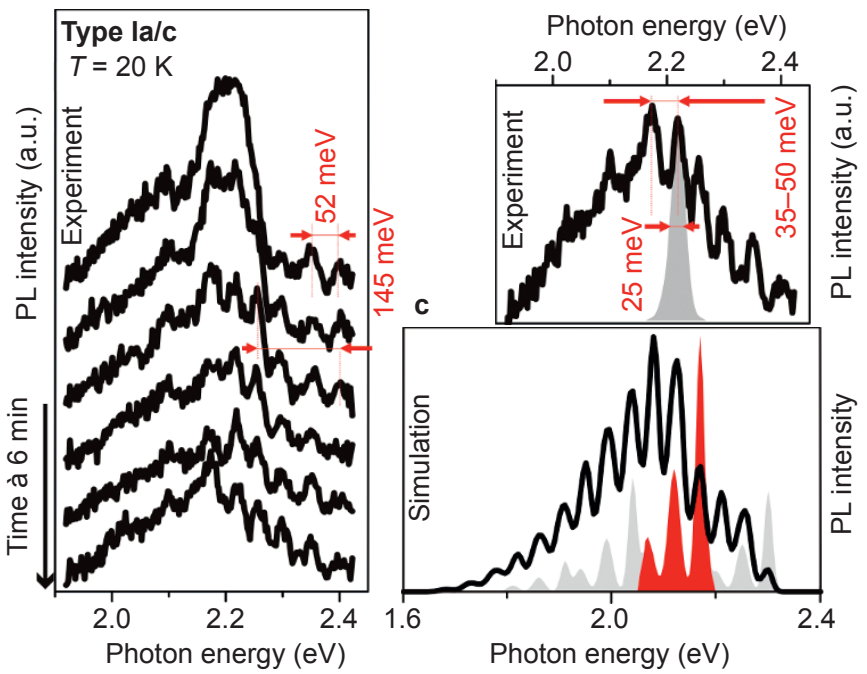

Figure 6 Influence of SD (a) A time series of consecutive single-NC spectra (offset for clarity) acquired at one specific spot; a transition between a type-la and type-Ic spectrum is evident. This series was measured at the same spot as the $20 \mathrm{~K}$ spectrum from Figure 4a, only with higher excitation power. (b) Average of the two lowermost spectra from panel a. (c) Simulated PL spectrum resulting from SD (black curve). The shaded gray curve is the input, "intrinsic" type-lb spectrum, which underwent simulated SD; its main trionic line, including phonon replicas, is highlighted in red for easier orientation. (Panels $\mathbf{b}$ and $\mathbf{c}$ are to scale in the photon-energy axis.)

(i) The observed peak spacing of the trionic structure and its temperature dependence (Figures $2 \mathrm{a}$ and $5 \mathrm{a}$ ) are indicative of the energetic distance of quantized hole levels in SiNCs ${ }^{34,35}$. The appearance of a low-energy replica spaced by quantization energy with decreasing amplitude toward lower emission energies can be naturally explained by the shake-up process within the radiative recombination of a positively charged trion, as sketched in Figure 1c (because of the decreasing amplitude, this process cannot be assigned to hot-carrier recombination).

(ii) The trion replicas share the same spectral sub-structure, as indicated in Figures 1e and 5c. This sub-structure originates from the participation of a core-related silicon phonon, which is a strong indication of the emission from intrinsic excitonic states. Similar coupling of the trion replicas to the same phonon mode was also observed in the trionic emission of II-VIbased NCs (see Figure S2 in the Supplementary Information of Ref. 16).

(iii) The observed high-energy wing is connected to the recombination of not completely relaxed holes, as sketched in Figure 1d. The observed behavior of the high-energy wing is in complete agreement with theoretical calculations of multiphonon carrier relaxation in $\mathrm{SiNCs}^{32}$, in stark contrast to a possible phonon-based explanation. In addition to slowed-down cooling of carriers for NCs of a suitable size, the hot carrier population is replenished by the presence of a second hole excited to a higher level during trionic recombination, which makes the observation of the hot-hole-related high-energy wing more probable.

(iv) The competing three-particle Auger recombination that is inherent to bulk silicon and was reported recently in "ordinary" indirect SiNCs (Auger lifetime 50-100 ps) ${ }^{41}$ cannot apply in the present case of direct-bandgap SiNCs because Auger recombination is known to be largely enhanced due to the specific features of silicon's indirect bandstructure ${ }^{42}$. However, because the bandstructure of the SiNCs under study bears a closer resemblance to direct-bandgap materials than it does to bulk Si (see Figure 1a), direct-bandgap materials are a better starting point for comparison. Recent experiments (these experiments were performed for negatively charged CdSe NCs; however, this is a systematic study dealing with NCs of various sizes; since no other such study exists for SiNCs, let alone the strain-engineered direct-bandgap SiNCs under study, we believe the comparison with negatively charged CdSe NCs to be the closest match) in CdSe $\mathrm{NCs}^{43}$ confirmed the Auger rate in NCs sized approximately $3 \mathrm{~nm}$ to be $1 / \tau_{A}=(5 \pm 2) \times 10^{8} \mathrm{~s}^{-1}$. Using our measured ensemble PL quantum yield $(\eta=0.2)$ and PL decay $(\tau=2 \mathrm{~ns})$, we can easily estimate the radiative rate $^{24} 1 / \tau_{r}=\eta / \tau=10^{8} \mathrm{~s}^{-1}$ and the upper bound of the non-radiative Auger rate $1 / \tau_{A}=1 / \tau-1 / \tau_{r}=$ $4 \times 10^{8} \mathrm{~s}^{-1}$ in the SiNCs under study. This is in excellent agreement with the Auger rate of CdSe NCs quoted above. Therefore, Auger recombination does not prevent the observation of radiative trionic recombination in this case.

(v) The observation of the light emission of positively charged trions even below the 1 exciton/NC threshold a priori requires the presence of charged NCs. However, the presence of substrate-deposited NCs charged for long time intervals (much longer than the radiative lifetime: microseconds to minutes) can be expected because charged NCs are also known to play an important role in blinking ${ }^{17,44,45}$, where practically identical types of samples are studied. In blinking experiments, it is also the electron, as a lighter particle, that is expected to escape the $\mathrm{NC}^{46}$.

Luminescing trions typically appear only in direct-bandgap semiconductors. (The only reference of trion luminescence in connection with indirect-bandgap semiconductors are, to the best of our knowledge, lineshape experiments in $\mathrm{Ge}^{47}$, which were later disproved ${ }^{48}$.) Therefore, the detection of bright trions actually provides an additional piece of evidence of the direct-bandgap nature of the SiNCs under study.

The observation of trionic emission obviously does not automatically imply the non-existence of common excitonic emission because excitonic emission would spectrally manifest itself in the same way as the main trionic peak. Therefore, the observed variability of the relative intensities of the first and second (trionic) peaks, evident from Supplementary Figs. S5b, S5c and S8a, can be a result of different efficiencies of the excitonic and trionic emission in different NCs and/or under slight fluctuations in the conditions of the local environment.

The emission of biexcitons is not expected to play a significant role in the SiNCs under investigation, primarily because they should appear only at higher excitation fluxes, but also because the density of hole states close to the top of the valence band is considerably higher compared with a single isolated electron level at the conduction-band minimum in the $\Gamma$ point. It is not excluded that biexciton luminescence may occur under slightly different experimental conditions. By contrast, non-radiative Auger recombination of four quasiparticles $(2 \mathrm{e}+2 \mathrm{~h})$ inside a small NC might prove very efficient and prevent any manifestation of PL of biexcitons. These issues, however, go beyond the scope of the present work. 
Although trionic emission clearly cannot be observed in (oxidepassivated) indirect-bandgap SiNCs with long radiative lifetimes $(>10 \mu \mathrm{s})$ due to competitive non-radiative Auger recombination (of the order of ns or less), it represents a feasible, interesting alternative explanation for structured single-NC spectra in SiNCs with short radiative lifetime $e^{4,6,23}$, especially because a correlation between the short radiative lifetime and structured spectra has been put forward ${ }^{23}$. However, such hypothesis would require further experiments in those samples to be verified, e.g., in the form of LT single-NC spectroscopy.

\section{CONCLUSION}

In conclusion, we provide an in-depth analysis of single-NC spectroscopy of strain-engineered direct-bandgap SiNCs at temperatures ranging from RT to $9 \mathrm{~K}$. Compared with the traditionally studied indirect-bandgap SiNCs, the strain-engineered NCs are characterized by significantly enhanced radiative rates (radiative lifetime $10 \mathrm{~ns}$ ) and, at the same time, by reduced non-radiative Auger rates (Auger lifetime $2.5 \mathrm{~ns}$ ). The representative spectral shape, which is only marginally influenced by spectral diffusion, consists of $2-3$ peaks approximately $150 \mathrm{meV}$ apart, each of which possesses a sub-structure of lines spaced by approximately $50 \mathrm{meV}$. The linewidth of the narrowest observed lines reaches $25.9 \mathrm{meV}$ at $\mathrm{RT}$ and $\leqslant 1 \mathrm{meV}$ at $20 \mathrm{~K}$. Whereas the substructure is assigned to Si phonon replicas in tensile-strained SiNCs, the $150-\mathrm{meV}$ spectral structure is argued to arise from the radiative recombination of positively charged trions. These findings serve as a strong additional support for the direct-bandgap nature of the SiNCs under study and, at the same time, broaden the class of quantum dots in which trionic emission has been observed.

\section{COMPETING INTERESTS}

The authors declare that they have no competing financial interests.

\section{ACKNOWLEDGEMENTS}

Czech Science Foundation Funding (Grant Nos. GPP204/12/P235 (Kateřina Kůsová) and P108/12/G108 (Ivan Pelant)) and a L'Oréal-UNESCO for Women in Science scholarship (Kateřina Kůsová) are gratefully acknowledged. Part of this work was supported by the Czech-Japan collaborative project LG14246 (Jan Valenta). The authors thank L. Ondič for help with the manuscript.

1 Hapala P, Kůsová K, Pelant I, Jelínek P. Theoretical analysis of electronic band structure of 2- to 3-nm Si nanocrystals. Phys Rev B 2013; 87: 195420.

2 Hannah DC, Yang J, Podsiadlo P, Chan MK, Demortiere A et al. On the origin of photoluminescence in silicon nanocrystals: pressure-dependent structural and optical studies. Nano Lett 2012; 12: 4200-4205.

3 Sangghaleh F, Bruhn B, Schmidt T, Linnros J. Exciton lifetime measurements on single silicon quantum dots. Nanotechnology 2013; 24: 225204

4 English DS, Pell LE, Yu Z, Barbara PF, Korgel BA. Size tunable visible luminescence from individual organic monolayer stabilized silicon nanocrystal quantum dots. Nano Lett 2002; 2: 681-685.

5 Kůsová K, Hapala P, Valenta J, Jelínek P, Cibulka $O$ et al. Direct bandgap silicon: tensile-strained silicon nanocrystals. Adv Mater Interf 2014; 1: 1300042.

6 Dohnalová K, Fučíková A, Umesh CP, Humpolíčková J, Paulusse JM et al. Microscopic origin of the fast blue-green luminescence of chemically synthesized non-oxidized silicon quantum dots. Small 2012; 8: 3185-3191.

7 Li Q, He Y, Chang J, Wang L, Chen $\mathrm{H}$ et al. Surface-modified silicon nanoparticles with ultrabright photoluminescence and single-exponential decay for nanoscale fluorescence lifetime imaging of temperature. J Am Chem Soc 2013; 135: 14924-14927.

8 Dohnalová K, Poddubny AN, Prokofiev AA, de Boer WDAM, Umesh CP et al. Surface brightens up Si quantum dots: direct bandgap-like size-tunable emission. Light-Sci Appl 2013; 2: e47; doi: 10.1038/lsa.2013.3.

9 Dohnalová K, Gregorkiewicz T, Kůsová K. Silicon quantum dots: surface matters. J Phys Condens Matter 2014; 26: 173201.

10 Poddubny AN, Dohnalová K. Direct band gap silicon quantum dots achieved via electronegative capping. Phys Rev B 2014; 90: 245439.

11 Empedocles SA, Neuhauser R, Shimizu K, Bawendi MG. Photoluminescence from single semiconductor nanostructures. Adv Mat 1999; 11: 1243-1256.
12 Fernée MJ, Tamarat $\mathrm{P}$, Lounis $\mathrm{B}$. Cryogenic single-nanocrystal spectroscopy: reading the spectral fingerprint of individual CdSe quantum dots. J Phys Chem Lett 2013; 4: 609-618.

13 Wang X, Ren X, Kahen K, Hahn MA, Rajeswaran M et al. Non-blinking semiconductor nanocrystals. Nature 2009; 459: 686-689.

14 García-Santamaría F, Chen Y, Vela J, Schaller RD, Hollingsworth JA. Suppressed Auger recombination in "giant" nanocrystals boosts optical gain performance. Nano Lett 2009; 9: 3482-3488.

15 Wang C, Wehrenberg BL, Woo CY, Guyot-Sionnest P. Light emission and amplification in charged CdSe quantum dots. J Phys Chem B 2004; 108: 9027-9031.

16 Fernée MJ, Littleton BN, Rubinsztein-Dunlop H. Detection of bright trion states using the fine structure emission of single CdSe/ZnS colloidal quantum dots. ACS Nano 2009; 3: 3762-3768.

17 Galland C, Ghosh Y, Steinbrück A, Sykora M, Hollingsworth JA et al. Two types of luminescence blinking revealed by spectroelectrochemistry of single quantum dots. Nature 2011; 479: 203-207.

18 Mason MD, Credo GM, Weston KD, Buratto SK. Luminescence of individual porous Si chromophores. Phys Rev Lett 1998; 80: 5405-5408.

19 Sirbuly DJ, Gargas DJ, Mason MD, Carson PJ, Buratto SK. Optical anisotropy in individual porous silicon nanoparticles containing multiple chromophores. ACS Nano 2008; 2: 1131-1136.

20 Valenta J, Fucikova A, Vácha F, Adamec F, Humpolíčková J et al. Light-emission performance of silicon nanocrystals deduced from single quantum dot spectroscopy. Adv Funct Mater 2008; 18: 2666-2672.

21 Valenta J, Juhasz R, Linnros J. Photoluminescence spectroscopy of single silicon quantum dots. Appl Phys Lett 2002; 80: 1070-1072.

22 Martin J, Cichos F, Huisken F, von Borczyskowski C. Electron-phonon coupling and localization of excitons in single silicon nanocrystals. Nano Lett 2008; 8: 656-660.

23 Schmidt T, Chizhik Al, Chizhik AM, Potrick K, Meixner AJ et al. Radiative exciton recombination and defect luminescence observed in single silicon nanocrystals. Phys $\operatorname{Rev} B$ 2012; 86: 125302.

24 Kůsová K, Cibulka O, Dohnalová K, Pelant I, Valenta J et al. Brightly luminescent organically capped silicon nanocrystals fabricated at room temperature and atmospheric pressure. ACS Nano 2010; 4: 4495-4504.

25 Sychugov I, Juhasz R, Valenta J, Linnros J. Narrow Luminescence Linewidth of a Silicon Quantum Dot. Phys. Rev. Lett 2005; 94: 087405.

26 Sychugov I, Valenta J, Mitsuishi K, Fujii M, Linnros J. Photoluminescence measurements of zero-phonon optical transitions in silicon nanocrystals. Phys Rev B 2011; 84: 125326.

27 Bruhn B, Valenta J, Sychugov I, Mitsuishi K, Linnros J. Transition from silicon nanowires to isolated quantum dots: optical and structural evolution. Phys Rev $B$ 2013; 87: 045404.

28 Dohnalová K, Ondič L, Kůsová K, Pelant I, Rehspringer JL et al. White-emitting oxidized silicon nanocrystals: discontinuity in spectral development with reducing size. J Appl Phys 2010; 107: 053102

29 Kůsová K, Cibulka O, Dohnalová K, Pelant I, Fučíková A et al. Yellow-emitting colloidal suspensions of silicon nanocrystals: fabrication technology, luminescence performance and application prospects. Physica E 2009; 41: 982-985.

30 Dohnalová K, Pelant I, Kůsová K, Gilliot P, Gallart M et al. Closely packed luminescent silicon nanocrystals in a distributed-feedback laser cavity. New J Phys 2008; 10: 063014.

31 Snoke DW, Shields AJ, Cardona M. Phonon-absorption recombination luminescence of room-temperature excitons in $\mathrm{Cu}_{2} \mathrm{O}$. Phys Rev B 1992; 45: 11693-11697.

32 Moskalenko AS, Berakdar J, Poddubny AN, Prokofiev AA, Yassievich IN et al. Multiphonon relaxation of moderately excited carriers in $\mathrm{Si} / \mathrm{SiO}_{2}$ nanocrystals. Phys Rev B 2012; 85: 085432.

33 Ondič L, Kůsová K, Ziegler M, Fekete L, Gärtnerová V et al. A complex study of the fast blue luminescence of oxidized silicon nanocrystals: the role of the core. Nanoscale 2014; 6: 3837-3845.

34 Moskalenko AS, Berakdar J, Prokofiev AA, Yassievich IN. Single-particle states in spherical $\mathrm{Si}_{2} \mathrm{SiO}_{2}$ quantum dots. Phys Rev B 2007; 76: 085427

35 Spenke E. Elektronische Halbleiter. Berlin Heidelberg: Springer; 1955.

36 Plakhotnik T, Fernée MJ, Littleton B, Rubinsztein-Dunlop H, Potzner C et al. Anomalous power laws of spectral diffusion in quantum dots: a connection to luminescence intermittency. Phys Rev Lett 2010; 105: 167402

37 Fernée MJ, Littleton B, Plakhotnik T, Rubinsztein-Dunlop H, Gómez DE et al. Charge hopping revealed by jitter correlations in the photoluminescence spectra of single CdSe nanocrystals. Phys Rev B 2010; 81: 155307.

38 Fernée MJ, Sinito C, Louyer Y, Tamarat $P$, Lounis B. The ultimate limit to the emission linewidth of single nanocrystals. Nanotechnology 2013; 24: 465703.

39 Gómez DE, Califano M, Mulvaney P. Optical properties of single semiconductor nanocrystals. Phys Chem Chem Phys 2006; 8: 4989-5011.

40 Pelant I, Valenta J. Luminescence Spectroscopy of Semiconductors. Oxford: Oxford University Press; 2012

41 Trinh MT, Limpens R, Gregorkiewicz T. Experimental investigations and modeling of Auger recombination in silicon nanocrystals. J Phys Chem C2013; 117: 5963-5968.

42 Abakumov VN, Perel VI, Yassievich IN. Nonradiative Recombination in Semiconductors. Amsterdam: North Holland; 1991.

43 Cohn AW, Rinehart JD, Schimpf AM, Weaver AL, Gamelin DR. Size dependence of negative trion Auger recombination in photodoped CdSe nanocrystals. Nano Lett 2014; 14: 353-358.

44 Frantsuzov $\mathrm{P}$, Kuno M, Jankó B, Marcus RA. Universal emission intermittency in quantum dots, nanorods and nanowires. Nature Phys 2008; 4: 519-522. 
45 Cichos F, von Borczyskowski C, Orrit M. Power-law intermittency of single emitters. Curr Opin Colloid Interface Sci 2007; 12: 272-284.

46 Efros AL, Rosen M. Random telegraph signal in the photoluminescence intensity of a single quantum dot. Phys Rev Lett 1997; 78: 1110-1113.

47 Thomas GA, Rice TM. Trions, molecules and excitons above the Mott density in Ge. Solid State Commun 1977; 23: 359-363.

48 Zhidkov AE, Pokrovskii YE. Existence of bi-excitons and trions in germanium. JETP Lett 1979; 30: 468-471.

(c) (1) (2) This work is licensed under a Creative Commons Attribution-

(c) ${ }_{\text {BY NC }}$ sA NonCommercial-ShareAlike 4.0 Unported License. The images or other third party material in this article are included in the article's Creative Commons license, unless indicated otherwise in the credit line; if the material is not included under the Creative Commons license, users will need to obtain permission from the license holder to reproduce the material. To view a copy of this license, visit http://creativecommons.org/ licenses/by-nc-sa/4.0/ 